(c) 2016 IEEE. Personal use of this material is permitted. Permission from IEEE must be obtained for all other uses, in any current or future media, including reprinting/republishing this material for advertising or promotional purposes, creating new collective works, for resale or redistribution to servers or lists, or reuse of any copyrighted component of this work in other works.

DOI: 10.1109/EuCAP.2016.7481148 


\title{
Investigation on a 77-GHz Broadside Vivaldi Antenna
}

\author{
Claudia Vasanelli, Hedi Meti, Christian Waldschmidt, \\ Ulm University, Institute of Microwave Engineering, 89081 Ulm, Germany \\ E-mail: claudia.vasanelli@uni-ulm.de
}

\begin{abstract}
Vivaldi antennas are excellent radiating elements that provide high gain and wide bandwidth. However, due to their end-fire radiation pattern they are not suited for the integration in printed circuit board (PCB) technology, if a broadside radiation is required. This paper presents a modified Vivaldi antenna loaded with a periodic arrangement of metallic strips that allows to achieve a broadside radiation pattern. Good agreement is obtained between the full-wave simulation results and the experimental data in terms of impedance matching, gain and radiation pattern.
\end{abstract}

Index Terms-Vivaldi antennas, millimiter-wave antennas, endfire antennas.

\section{INTRODUCTION}

Radar systems are currently one of the key sensors of driver assistance systems [1]. The $76 \mathrm{GHz}-81 \mathrm{GHz}$ frequency band has been allocated in Europe for automotive radar systems.

The antenna is an integral part of the sensor and it has a great impact on the system performance. The specific application requires an antenna radiation pattern with a narrow beam in the elevation plane and a wide beam in the azimuth plane [2]. Moreover, standard PCB technology is mainly employed to achieve a cheap mass production of the sensors. Lightweight planar antenna elements allow the design of compact radar systems that could be easily integrated beyond the bumper of the car. Finally, an antenna with a broadband behavior helps to increase the robustness of the design against the unavoidable etching tolerances that can become critical at millimeter-wave frequencies.

Vivaldi antennas [3] are usually excellent planar radiating elements that present moderately high gain, wide bandwidth, and easy fabrication by means of standard PCB photo-etching process, but they are not suited for automotive radar applications due to their end-fire radiation pattern that increases the overall installation depth of the sensor. A broadside pattern is thus an appealing feature in the antenna design for automotive radar applications. In [2] the authors suggest the use of parabolic reflectors integrated on the metal housing of the radar sensor to achieve a broadside pattern from an end-fire antenna. This solution increases of course the dimensions and the weight of the sensor.

Recently, new designs of the Vivaldi antenna have been proposed to get a broadside radiation pattern [4], [5]. In [5] the authors describe a two-element parallel-fed array of Vivaldi antennas on a complex three-layers structure for the integration with a metal reflector, while in [4] for the first time the authors

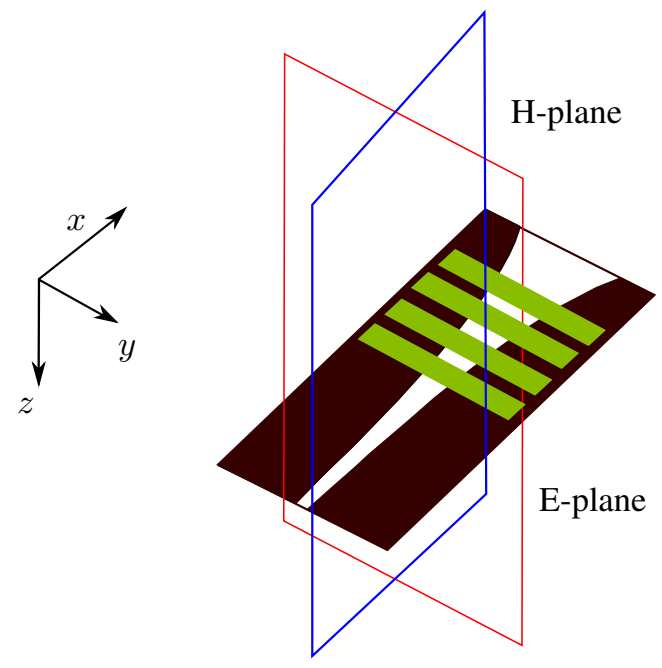

Fig. 1. Proposed antenna concept. The two main cuts of the radiation pattern are highlighted.

propose the use of periodic metallic strips placed beyond the antenna on a single layer structure to get an almost perfect broadside radiation at $2.45 \mathrm{GHz}$.

In this paper, the idea suggested in [4] is further developed for the first time at millimeter-wave frequencies to get a modified Vivaldi antenna with a broadside radiation pattern while keeping the aforementioned advantages of the original Vivaldi antenna.

\section{Antenna CONCEPT}

The antenna concept is shown in Fig. 1. A single-layer design is employed. On one side of the substrate, a Vivaldi antenna is placed, while on the opposite side, a periodic set of metallic strips is located beyond the taper of the antenna. The presence of the metallic strips allows an almost broadside radiation to take place.

The modified Vivaldi antenna can be basically divided into two main sections, that are highlighted in Fig. 2: the first one $\left(A-A^{I}\right)$ includes the distance from the slotline feeding position until the first metallic strip, and the second section $\left(\mathrm{A}^{\mathrm{I}}-\mathrm{A}^{\mathrm{II}}\right)$ includes the four strips up to the end of the taper of the antenna.

The first section is the same as a conventional end-fire Vivaldi antenna in terms of field distribution and wave prop- 


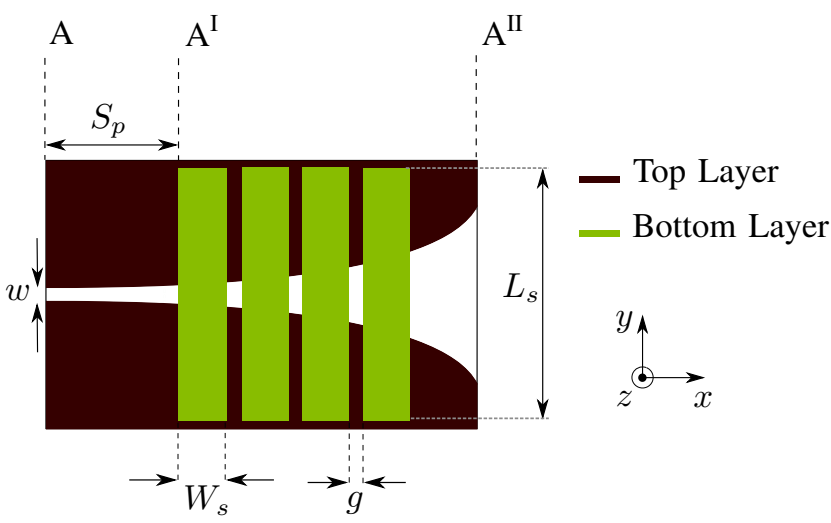

Fig. 2. 2D model of the radiating element

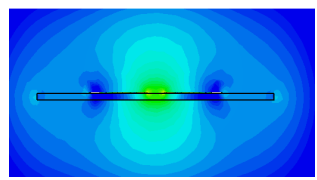

(a)

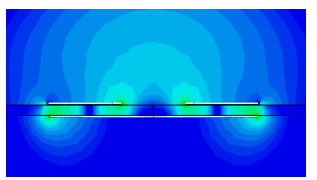

(b)

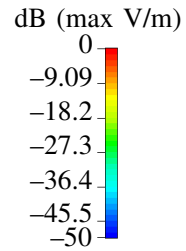

Fig. 3. Magnitude of the E-field on the cross sectional plane of (a) a conventional Vivaldi antenna and (b) the new modified Vivaldi antenna.

agation. The fundamental slotline mode propagates and the interaction with the periodic strips is still weak.

In the second section, by loading the back side of the substrate with the periodic copper strips, the field distribution changes. The structure is similar to a conductor-backed slotline [6], in which different modes propagate: the fundamental odd-mode and two parallel plate modes (in the two halves of the structure respectively) between the top metallization of the Vivaldi antenna and the metal strips on the back. As expected, the fundamental mode can radiate in the free space when it is not sufficiently bounded to the structure. This causes the main radiation mechanism of the antenna. On the other hand, the parallel plate mode propagates in the structure laterally until the open end of the transmission line. There the wave will be partially radiated and partially reflected back, where it will superimpose to the incident wave causing thus a standing wave pattern. Due to the discontinuity of the slot, the back-reflected wave will radiate, too.

Since the gap between the metallic strips is much smaller than the free-space wavelength at $77 \mathrm{GHz}\left(g \ll \lambda_{0}\right.$, see Tab. I), the set of metallic strips behaves indeed as a reflector for the antenna, causing thus the radiation only in the upper hemisphere, i.e. a broadside radiation pattern is obtained. This can be clearly seen in Fig. 3, where it is possible to compare the pattern of the magnitude of the E-field of a traditional Vivaldi antenna and the pattern of the new proposed structure. The plot confirms that the antenna radiates now in the upper hemisphere.

All the simulations have been carried out with a commercial full-wave simulation software. Table I reports the dimensions
TABLE I

MAIN PARAMETERS FOR THE DESIGN

\begin{tabular}{c||c}
\hline$S_{p}$ & $0.89 \mathrm{~mm}$ \\
\hline$W_{s}$ & $0.45 \mathrm{~mm}$ \\
\hline$L_{s}$ & $2.52 \mathrm{~mm}$ \\
\hline$g$ & $0.10 \mathrm{~mm}$ \\
\hline$w$ & $0.10 \mathrm{~mm}$ \\
\hline
\end{tabular}

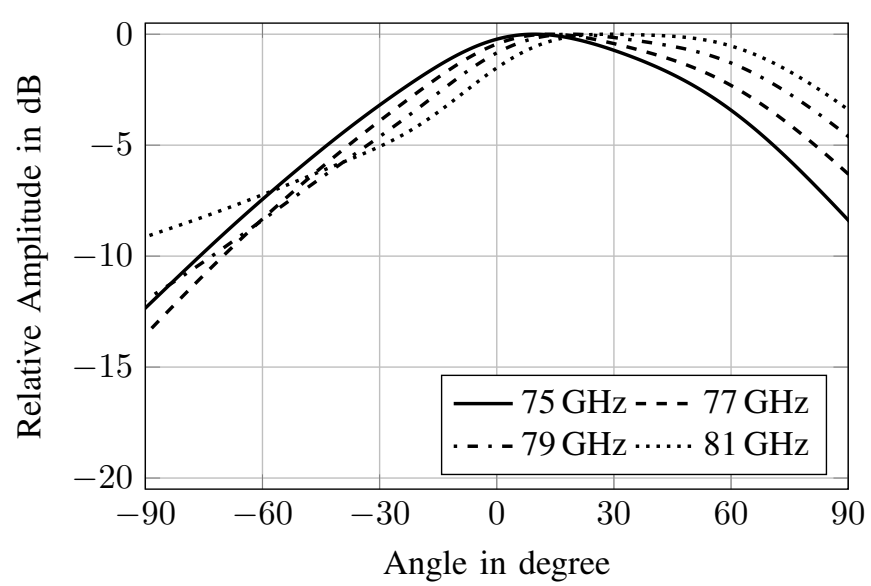

Fig. 4. Simulated radiation diagrams in the H-plane. The nearly broadside behavior is apparent.

of the main parameters in the design. The total length of the antenna is $3.7 \mathrm{~mm}$, while the total width is $2.6 \mathrm{~mm}$.

As shown in Fig. 4, the simulation results indicate that the radiation pattern is nearly broadside, because the direction of the main beam is slightly tilted from the boresight direction. As can be seen from Fig. 4, the tilt in the H-plane is not constant versus frequency and it increases from approximately $10^{\circ}$ at $75 \mathrm{GHz}$ to more than $30^{\circ}$ at $81 \mathrm{GHz}$. Due to the symmetry of the structure, no tilt of the main beam is expected in the E-plane.

Later investigations proved that the beam squinting is mainly related to the geometry of the taper. Indeed, by using a constant-width slot antenna, similar to what was presented in [8] with the same set of metallic strips on the bottom side of the substrate, the squinting is highly reduced. An almost constant tilt from $75 \mathrm{GHz}$ to $81 \mathrm{GHz}$ is thus observed, as it can be seen in Fig. 5. This additional property of the structure could be further exploited in a successive design of the proposed radiating element to control the tilt of the beam.

Also a nearly broadside radiation pattern can be clearly obtained also by using a metal plane instead of the set of metallic strips. This alternative design has also been examined. Although similar results are obtained in terms of impedance matching, the simulations showed that the tilt of the main beam related to the broadside radiation is significantly increased.

\section{Measurement Results}

A prototype of the proposed antenna has been fabricated with standard photo-etching process. The manufactured prototype can be seen in Fig. 6. The single layer structure is 


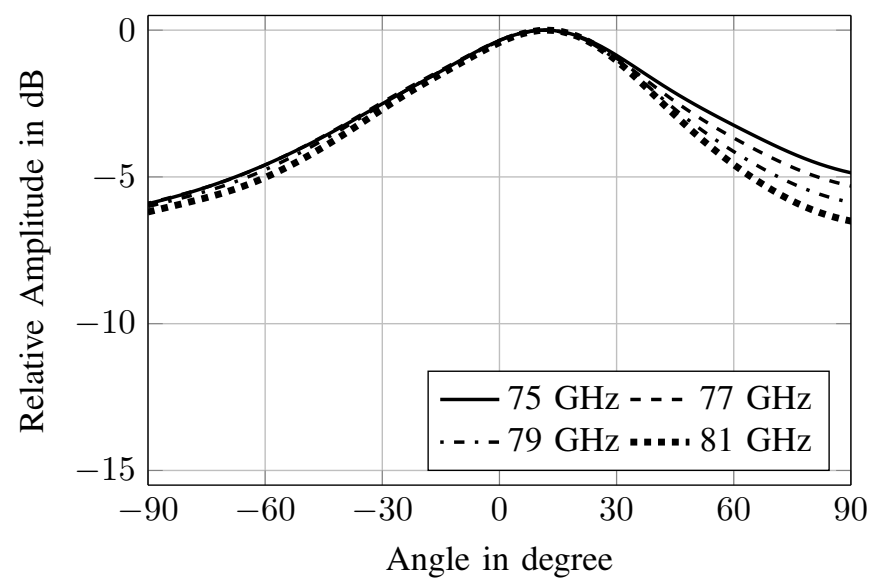

Fig. 5. Simulated radiation diagrams in the H-plane of the constant-width slot antenna. The direction of the main beam is stable versus frequency.

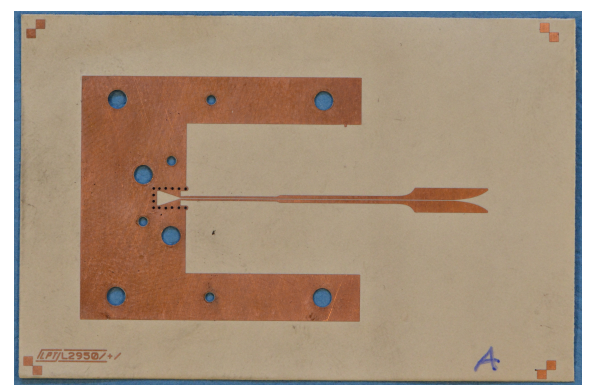

(a)

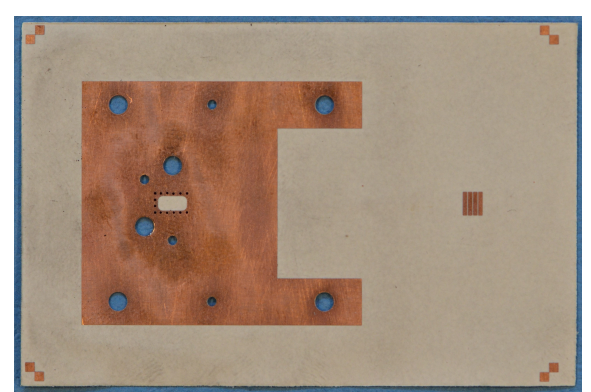

(b)

Fig. 6. (a) Top and (b) bottom view of the fabricated antenna

designed on a $127 \mu$ m-thick substrate (RO3003) from Rogers Corporation [7] with the relative permittivity $\varepsilon_{r}=3$. The visual inspection showed roughly $20 \mu \mathrm{m}$ etching tolerances.

\section{A. Waveguide Transition}

As can be seen from the picture of the fabricated protype in Fig. 6, for measurement purposes the antenna has been integrated with a transition from rectangular waveguide (WR12) to slotline.

A more detailed view of the transition is depicted in Fig. 7. First, a transition from waveguide to differential microstrip line (DMSL) is employed. The design is based on the transition presented in [9] and [10]. This transition transforms the $\mathrm{TE}_{10}$ mode of the rectangular waveguide into the odd mode of the differential microstrip line by suppressing the even mode.

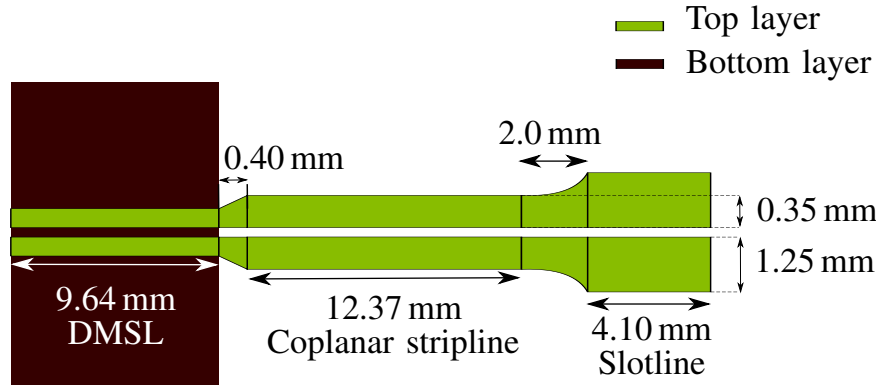

Fig. 7. Model of the waveguide-to-slotline transition. The gap between the lines is constant and it amounts to $0.10 \mathrm{~mm}$. The width of the DMSL is $0.19 \mathrm{~mm}$.

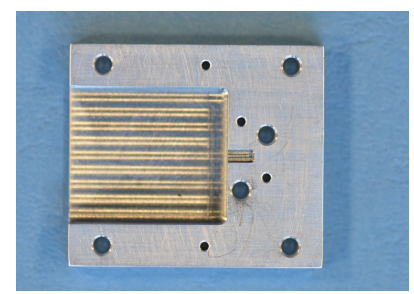

(a)

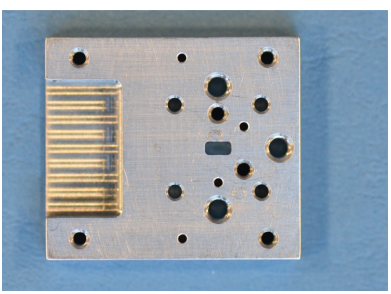

(b)
Fig. 8. (a) Backshort and (b) WR12 connector for the rectangular waveguide transition.

In the second part of the transition, the odd mode of the DMSL is then transformed through a coplanar stripline in the slotline mode, which is needed to feed the Vivaldi antenna. The aforementioned steps are clearly highlighted in the model of Fig. 7.

The PCB is perpendicularly inserted in the rectangular waveguide, which is then terminated on the opposite side by a backshort. The mechanical components that are used in the transition are depicted in Fig. 8. As can be seen in the pictures, both components have been extended to partially cover the DMSL with a hollow in the metallic mount. This solution reduces spurious radiation from the feeding and gives mechanical stability to the thin substrate.

\section{B. Reflection Coefficient}

First, the reflection coefficient has been measured. The comparison between the simulated and measured $S_{11}$ is reported in Fig. 9. A good agreement is obtained. The simulation result refers to the relative permittivity of $\varepsilon_{r}=3.1$ of the substrate. The used substrate is indeed not characterized at millimeterwave frequencies by the manufacturer and small differences in the value of the permittivity are therefore possible.

By means of time domain gating, the effect of the waveguide transition has been removed and the reference plane has been shifted to the beginning of the slotline. The comparison between simulation and measurement is depicted in Fig. 10. As can be seen, the impedance matching is perfectly achieved in the frequency range of interest. 


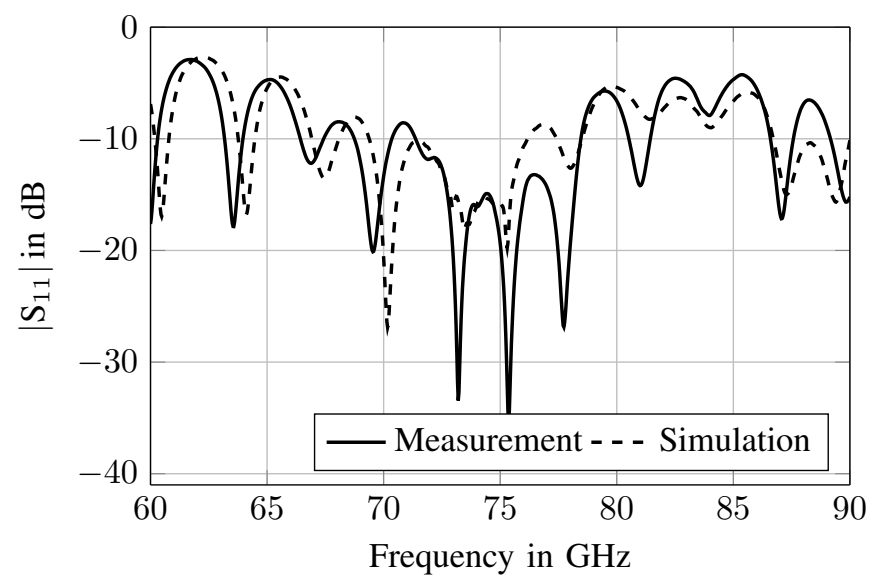

Fig. 9. Measured and simulated reflection coefficient of the proposed antenna integrated with the waveguide-to-slotline transition.

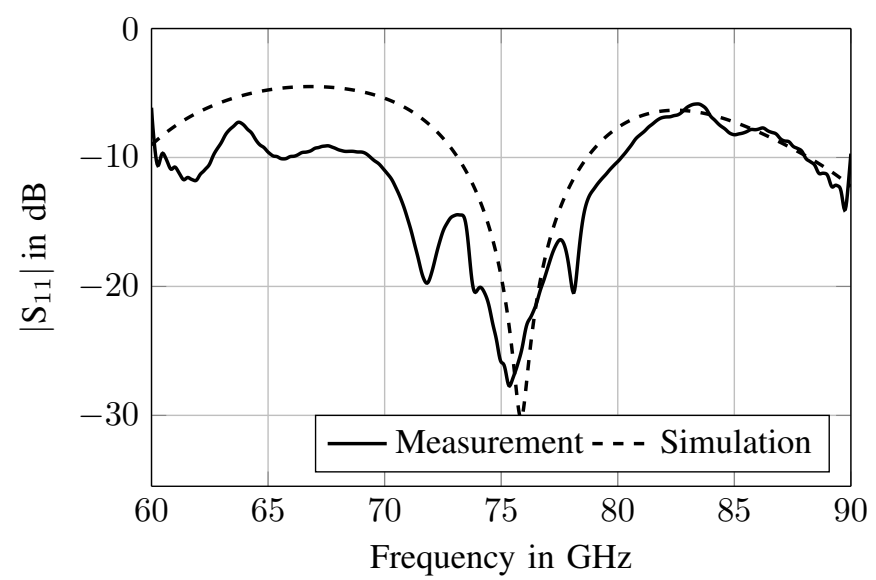

Fig. 10. Measured and simulated reflection coefficient of the proposed antenna. The effect of the transition has been removed by means of time gating.

\section{Radiation Pattern and Gain}

Afterwards, the radiation pattern of the antenna has been measured in the E- and H-plane. During the measurement in an anechoic chamber (see Fig. 11), the metallic cap of the transition and the frequency converter module have been covered with absorber material to minimize unwanted reflections.

The measurement results at $77 \mathrm{GHz}$ are shown in Fig. 12 . When the Vivaldi antenna is integrated with the waveguide transition, significant ripples appear in the radiation pattern. Although the nearly broadside radiation of the antenna is still maintained, the frequency squint behavior can not be evaluated anymore. Similar results are obtained at other frequency points in the bandwidth of interest, but they are not reported in this paper to minimize duplication.

Finally, the gain of the fabricated antenna has been measured using a 25-dB standard gain horn antenna and then compared to the simulated values. As can be seen in Fig. 13, in the frequency range from $75 \mathrm{GHz}$ to $81 \mathrm{GHz}$ a good agreement has been achieved with differences between the

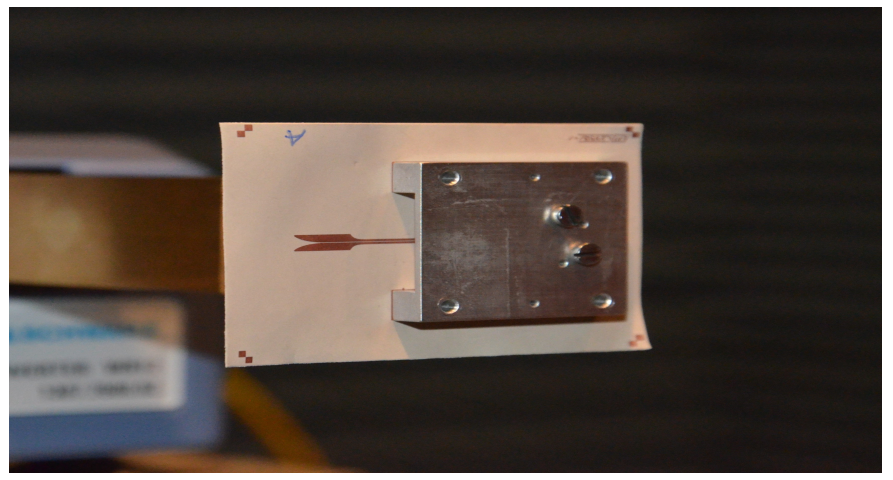

Fig. 11. Photo of the antenna in the anechoic chamber. The picture has been taken before placing the absorber material.

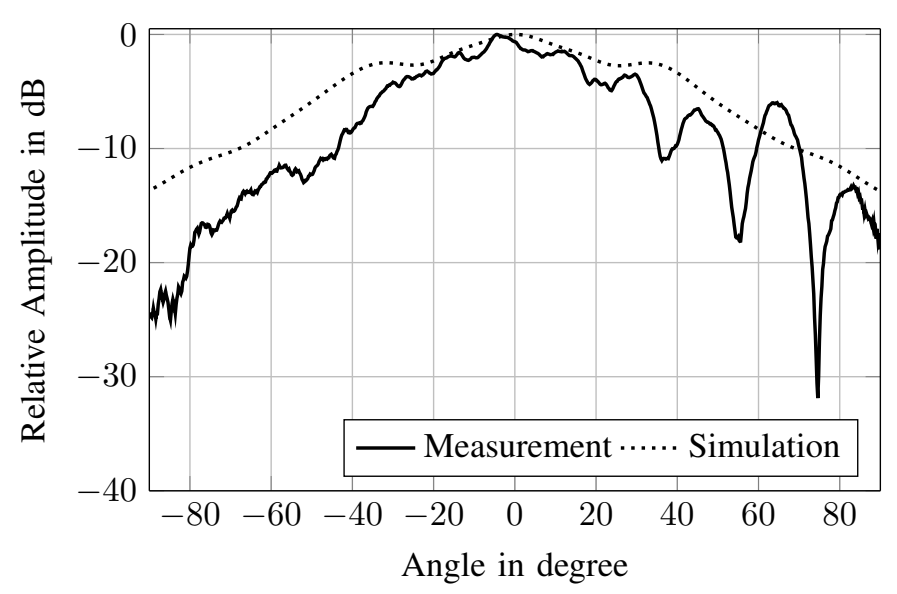

(a)

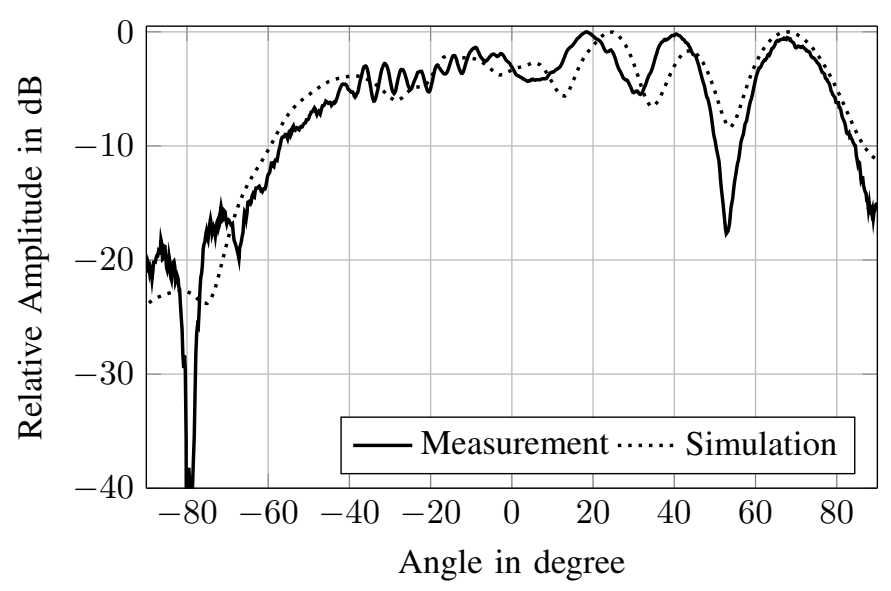

(b)

Fig. 12. Measured and simulated radiation pattern at $77 \mathrm{GHz}$ of the proposed antenna in the (a) E-plane and (b) H-plane.

numerical and experimental data within $1 \mathrm{~dB}$. The differences in the measured and simulated gain could be mainly due to fabrication inaccuracies. 


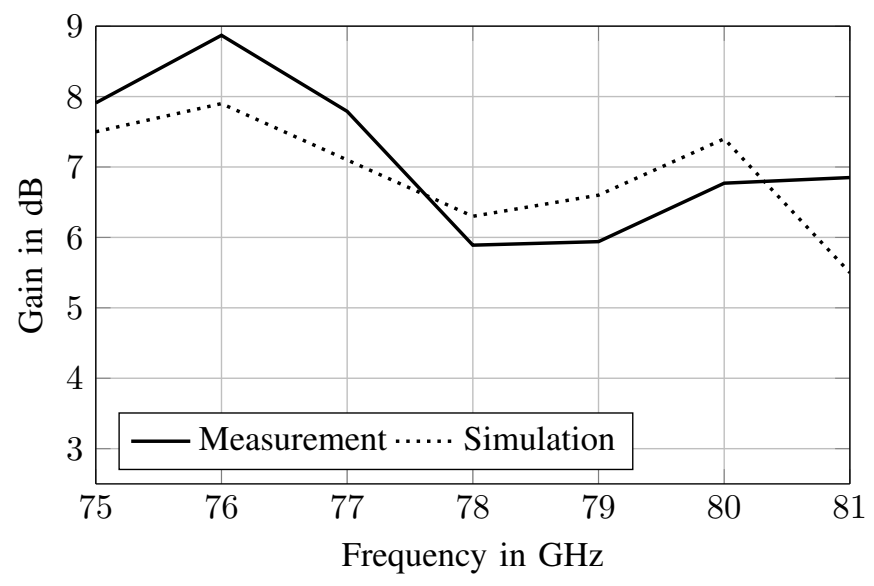

Fig. 13. Measured and simulated gain of the modified Vivaldi antenna at boresight.

\section{CONCLUSION}

In this paper, for the first time a modified Vivaldi antenna with broadside radiation pattern has been presented at millimeter-wave frequencies. It has been proved that by loading the back side of a Vivaldi antenna with a periodic set of metallic stripes it is possible to get a nearly broadside radiation pattern.

The measurement results of both the reflection coefficient and the radiation pattern are in good agreement with the numerical results. The measured gain is also close to the value predicted by the full-wave simulations in the frequency range of interest.

\section{ACKNOWLEDGMENT}

The authors would like to thank Dr.-Ing. Frank Bögelsack for the helpful suggestions about the farfield measurements.

\section{REFERENCES}

[1] J. Hasch, E. Topak, R. Schnabel, T. Zwick, R. Weigel, and C. Waldschmidt, A Millimeter-Wave Technology for Automotive Radar Sensors in the $77 \mathrm{GHz}$ Frequency Band, IEEE Trans. Microwave Theory Tech., vol. 60, no. 3, pp. 845-860, 2012.

[2] S. Beer, G. Adamiuk, and T. Zwick, Novel Antenna Concept for Compact Millimeter-Wave Automotive Radar Sensors, IEEE Antennas Wireless Propagat. Lett., vol. 8, pp. 771-774, 2009.

[3] P.J. Gibson, The Vivaldi Aerial, in Proc. of the 9th European Microwave Conf., 1979, pp.101-105.

[4] F. Congedo, G. Monti, L. Tarricone, and V. Bella, A $2.45 \mathrm{GHz}$ Vivaldi Rectenna for the Remote Activation of an End Device Radio Node, in IEEE Sensors Journal, vol. 13, no. 9, pp. 3454-3461, 2013.

[5] R. Hahnel, and D. Plettemeier, $60 \mathrm{GHz}$ Broadside Radiating Vivaldi Antenna, in IEEE Antennas and Propagation Society Int. Symposium (APSURSI), 2013, pp.1832-1833.

[6] J. Zehentner, J. Machac, J. Mrkvica, and C. Tuzi, The Inverted ConductorBacked Slotline - a Challenge to Antenna and Circuit Design, in Proc. of the 33rd European Microwave Conf., 2003, pp. 73-76.

[7] Rogers Corporation, CT 06263, USA.

[8] W.K. Ofosu, and D. Mirshekar-Syahkal, Radiation Pattern of Short Constant-Width Slot Antenna, in Electronics Letters, vol. 31, no. 1, pp. 7-8, 1995.

[9] Z. Tong, A. Stelzer, W. Menzel, C. Wagner, R. Feger, and E. Kolmhofer, A Wide Band Transition from Waveguide to Differential Microstrip Lines, in Asia-Pacific Microwave Conf. (APMC), 2008, pp. 1-4.
[10] M. Frei, F. Bauer, W. Menzel, A. Stelzer, and L. Maurer A $79 \mathrm{GHz}$ Differentially Fed Grid Array Antenna, in Proc. of the 41st European Microwave Conf. (EuMC), 2011, pp. 1320-1323. 